\title{
Numerical Study of Nonlinear Self-sustained Thermoacoustic Instability in a Swirling Combustor
}

\author{
Yuze Sun and Dan Zhao* \\ Department of Mechanical Engineering, University of Canterbury, Christchurch 8041, New Zealand
}

\begin{abstract}
Self-exited thermoacoustic instability is undesirable in gas turbines and other propulsion systems due to structural vibration, overheating and flame flashback. Such instability is characterized with large-amplitude limit cycle oscillations. It is typically resulting from the dynamic interaction between turbulence flow-flame-acoustics. A better understanding on the interaction, and an alternative design of effective damping/control approach to stabilize combustors are wanted. In this work, 3D numerical simulations are conducted on a swirling combustor to gain insight on the generation of nonlinear swirling flow-flame-coupled thermoacoustic instability. To capture the turbulence flow-combustion-acoustics interaction, the classical turbulence model for swirling flow RNG $k-\varepsilon$ and eddy dissipation concept model with 2-steps are applied. The model is validated first with the experimental data available in the literature. Then it is applied to study the effect of swirling number $S_{\mathrm{N}}$. It is found that increasing the $S_{\mathrm{N}}$ leads to the amplitude of combustion instability being increased. The dominant frequency is found to shift to a higher value with increased $S_{N}$. To attenuate the selfexcited thermoacoustic instability, we propose an alternative passive control approach by implementing a heat exchanger at a pre-selected segment of combustor wall; its temperature $T_{H}$ could be varied. Numerical results show that properly setting $T_{H}$ can attenuate the thermoacoustic instability by approximately $20 \mathrm{~dB}$. The present study contributes to developing a platform to simulate nonlinear thermoacoustic instability in a swirling combustor. It also opens up an alternative control means to prevent the onset or attenuate the undesirable limit cycle oscillations.
\end{abstract}

\section{Introduction}

L EAN premixed swirling combustion is a popular combustion technology to reduce chemical emission and enhance combustion [1]. However, such combustion technology is associated with undesirable combustion instability [2]. It is characterized with large amplitude acoustic oscillations, (also known as limit cycle oscillations) and may lead to structural vibration, and overheating problem [3].

Swirling flows can help improve fuel-air mixing, stabilize the flame and reduce the combustion length. As these merits, most industry gas turbines adopt swirling flow for efficient and clean combustion [4]. Intensive numerical research has been conducted on swirl-involved combustor to capture the flow field characteristics and investigate flow-flame interaction on thermoacoustic instability. Huang et al. [5] investigated a lean-premixed swirl-stabilized combustor by using large eddy simulations (LES). Results show that swirling intensities determines the pressure and heat release fluctuations. Yellugari et al. [6] conducted RANS numerical simulation with flamelet generated manifold (FGM) combustion model to investigate the non-reacting and reacting flows with and without the central rod at different swirling intensities on a swirling burner. The central toroidal recirculation zone (CTRZ) moved upstream along with the flame with the increase in the swirl number. Mansouri et al. [7] studied flow behavior and flame stability of premixed propane swirling flames by using RANS simulation. Results show in such combustor, the swirl number reaches a critical value an inner recirculation zone (IRZ) appears when the swirl number reaches a critical value 0.75 . And large swirling intensities may lead to the phenomenon of the flame flashback.

Thermoacoustic instabilities may be mitigated by active control or passive control [8]. Active control method are typically applied by applying a dynamic actuator such as a loudspeaker. However, appropriate control parameters such as frequency and amplitude of external signal have to be verified by experiments which limits the effectiveness [9]. Passive

*Corresponding author. Email: dan.zhao@ canterbury.ac.nz (Dan Zhao), AIAA Associate Fellow.

1

American Institute of Aeronautics and Astronautics 
control techniques generally involves modifying the structure of combustor by installing acoustic damper such as perforated liners or resonators. However, modifying the combustion system can be high-cost, inefficient and lack of flexibility [10]. Recently, alternative methods have recently been proposed and investigated besides the classical active and passive control methods. For example, Wu et al. [11] experimentally demonstrated thermoacoustic instability could be controlled by using an electrical heater in a traditional Rijke tube and a Y-shaped Rijke tube.

The present work focuses on the numerical investigation of nonlinear self-sustained thermoacoustic instabilities in a swirling combustor. The structure of the paper is as follows. Section II describes the computation domain, mesh generation and numerical methods used and operation conditions. The numerical validations results and effects of swirling number $S_{N}$ and temperature of heat exchanger $\mathrm{T}_{\mathrm{H}}$ on thermoacoustic instabilities excited by swirling flame are discussed in Section III. Finally, the content of this paper are summarized and concluded in section IV.

\section{Model and Numerical Method}

\section{A. Geometry and Grid generation}

The physical model of the premixed swirling combustor is a simplified gas turbine swirling type combustor base on our previous experimental rig [12]. Computational domain and previous experimental rig are shown in Fig. 1(a). The combustor length $\left(L_{\mathrm{c}}\right)$ and radius $\left(R_{\mathrm{c}}\right)$ are $700 \mathrm{~mm}$ and $50 \mathrm{~mm}$. Moreover the combustor inlet is an annular with $4 \mathrm{~mm}$ inner radius $R_{i}$ and $11 \mathrm{~mm}$ outer radius $R_{\mathrm{s}}$, respectively. The length of heat exchanger $L_{\mathrm{h}}$ is $100 \mathrm{~mm}$ and heat exchanger are located at $L_{\mathrm{d}}=300 \mathrm{~mm}$ from the combustor outlet. In order to minimize the computational expenses, details of the swirler are not included in the numerical simulations. Moreover, a 1/8th segment of the combustor with rotational periodic boundary conditions is used to perform the simulations. 3D structured grids generation is shown in Fig. 1(b). Total grid number is $0.5 \mathrm{M}$ cells. In the region where flame is located, grids are refined for better prediction of the combustion and gradients.
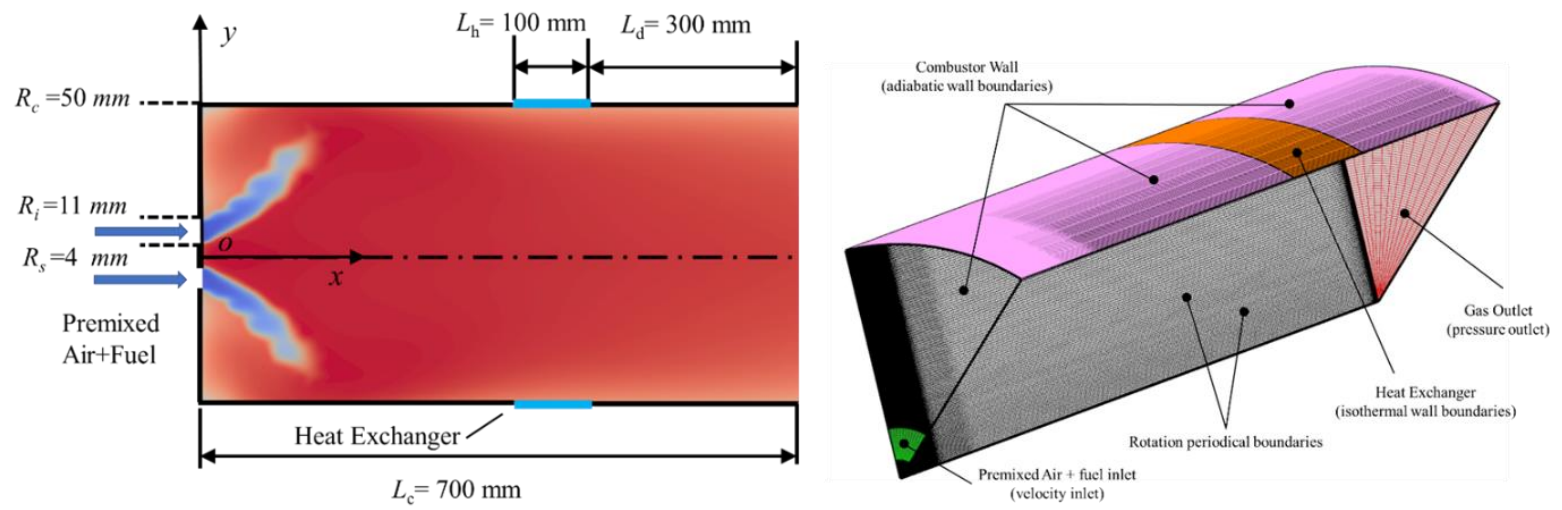

Fig. 1: (a) Geometrical details of the combustor (b) Grid topology of the 1/8th segment combustor.

\section{B. Numerical set up}

Reynolds-averaged Navier-Stokes (RANS) method is applied to solve the compressible equation of mass, momentum, energy, and species transport conservation. A RNG $k-\varepsilon$ turbulence model is adopted for prediction of turbulence swirling flow. The COUPLE algorithm scheme was used to handle the pressure-velocity coupling. And a transient solver with a fixed time-step of $1 \times 10^{-4} \mathrm{sec}$ is applied. Eddy-dissipation-concept model with a reduced 2-steps global mechanism is applied to model the chemistry reaction. The least squares cell based format is utilized as the gradient spatial discretization. The pressure spatial discretization format is the second order. And third-order MUSCL scheme is used for all equations, except the turbulence dissipations which are second-order upwind format. In addition, all numerical conditions were conducted by using the commercial CFD software, ANSYS Fluent 19.1.

The inlet boundary condition is velocity inlet corresponding an air flow rate of $180 \mathrm{~L} / \mathrm{min}$ at a temperature of 300 $\mathrm{K}$ with an $8 \%$ turbulence intensity. The equivalence ratio is fixed at $\Phi=0.9$. Axial and tangential velocity profiles are given at velocity inlet to generate a swirling flow. Swirling intensity is characterized by a non-dimension swirling number which can be calculated as [13]: 


$$
S_{N}=\frac{\int_{R_{S}}^{R_{i}} u_{a} u_{\theta} r^{2} d r}{R_{i} \int_{R_{S}}^{R_{i}} u_{a}^{2} r d r}
$$

where $R_{\mathrm{s}}$ and $R_{i}$ are the diameter of the centre shaft and the inlet; $u_{a}$ and $u_{\theta}$ are axial and tangential velocity.

\section{Results and Discussions}

\section{A. Validation}

To assess the effect of grid number on simulation results, three different meshes with $0.1 \mathrm{M}$ cells, $0.5 \mathrm{M}$ cells and $1 \mathrm{M}$ cells are selected to perform grid sensitivity studies. Fig. 2 shows axial velocity and temperature radial distributions at $x / R_{\mathrm{c}}=0.5$. The results show that grid with $0.1 \mathrm{M}$ cells is too coarse, while the profiles of grid with $0.5 \mathrm{M}$ and $1.0 \mathrm{M}$ cells are almost identical, which indicates that $0.5 \mathrm{M}$ cells are enough for present study. Mean velocity profiles obtained from simulation are compared with the experimental data from Taamallah et al. [14]. The velocity profiles of two different locations are shown in Fig. 3. The overall agreement is reasonable although some deviations can be observed.
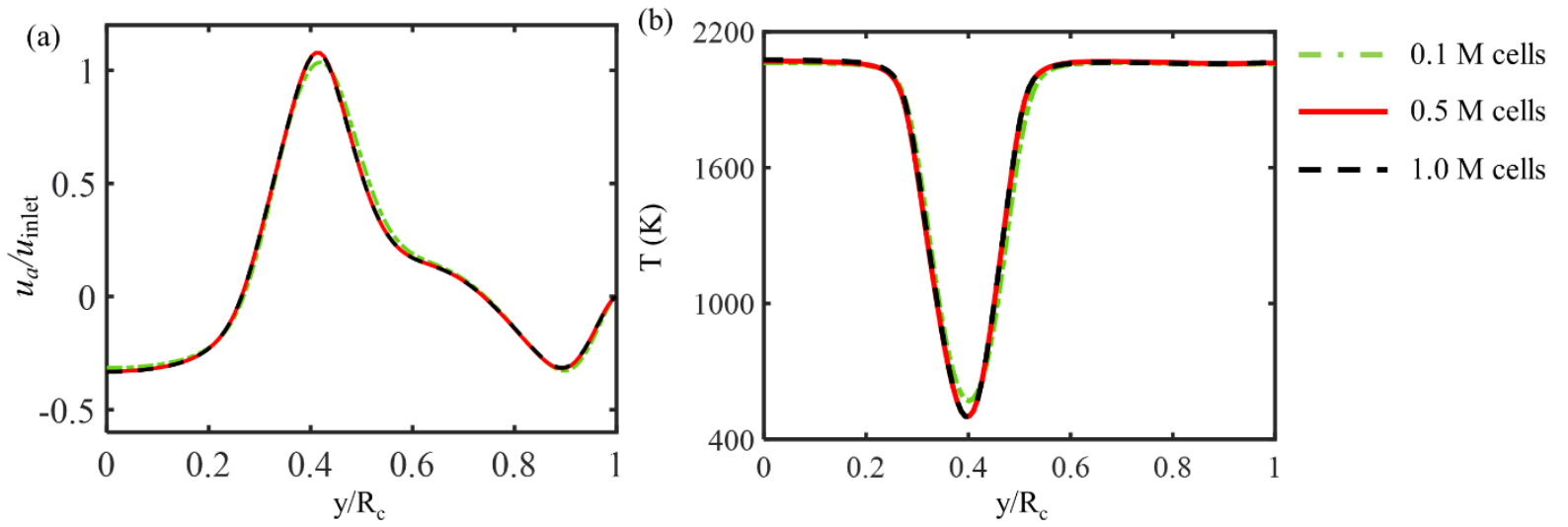

Fig. 2 Mesh independence study: (a) normalized axial velocity profiles (b) temperature profiles at $x / \mathbf{R}_{c}=0.5$

\section{Simulation $\longrightarrow$ Exp.}

(a)
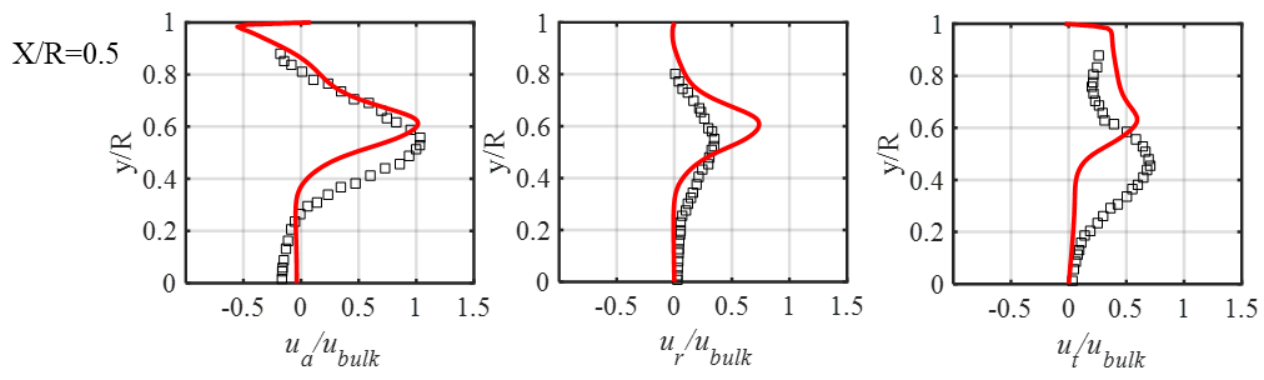

(b) $\mathrm{X} / \mathrm{R}=1.5$
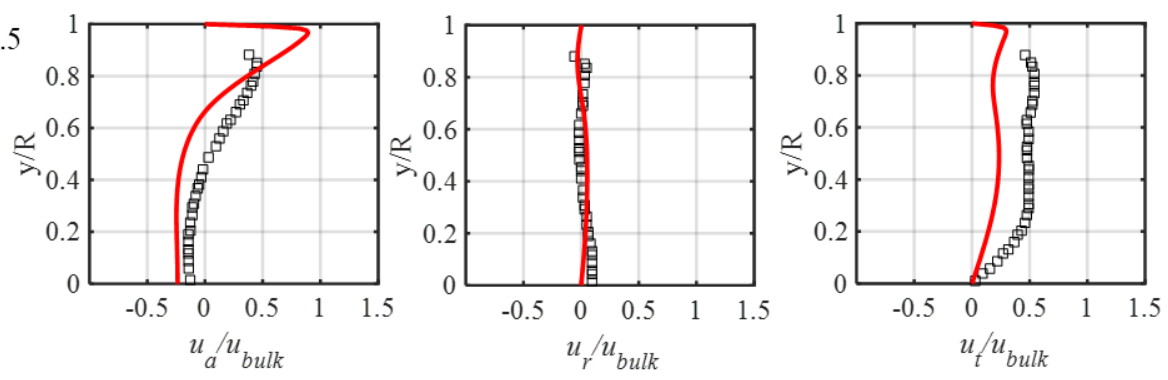

3

American Institute of Aeronautics and Astronautics 
Fig. 3: Validation results: mean axial (left), radial (center) and tangential (right) velocity profiles at different cross sections (a) $x / R=0.5$ and (b) $x / R=1.5$ of the combustor.

\section{B. Effect of swirling number}

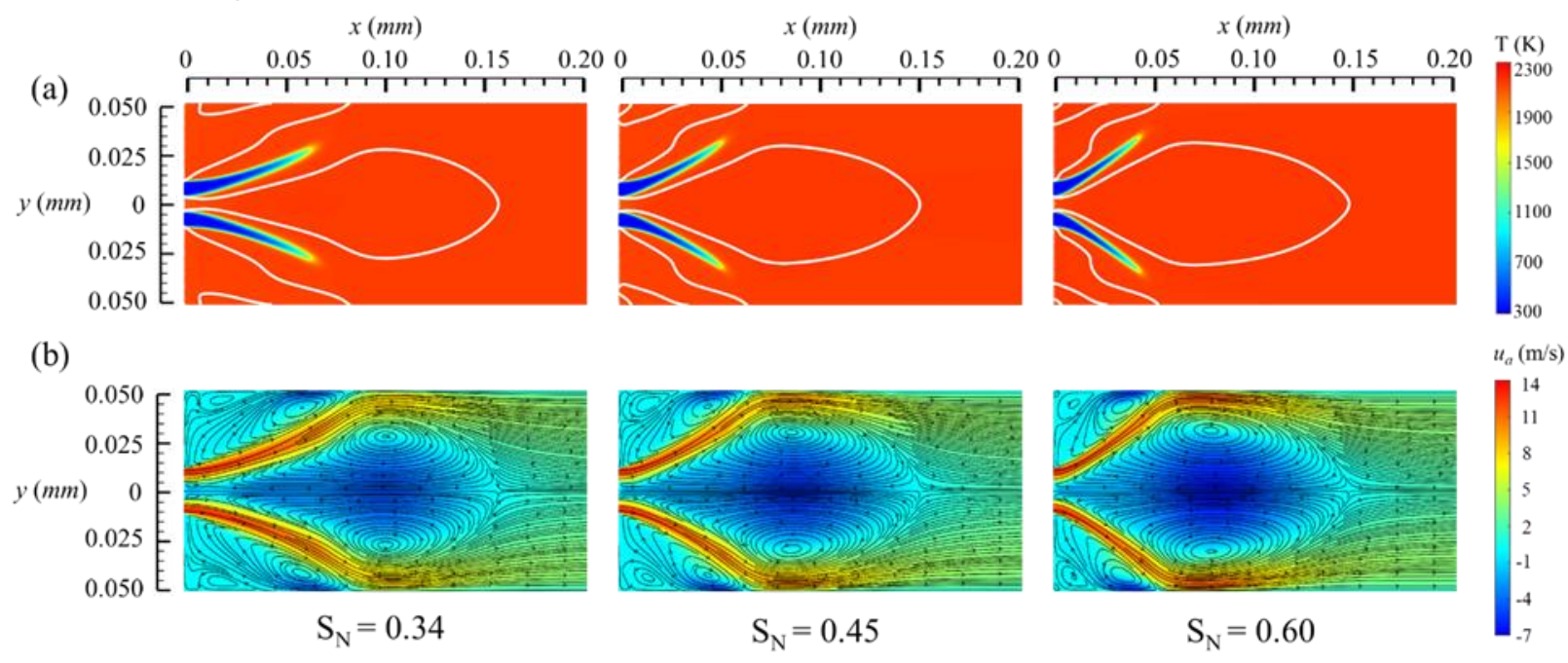

Fig. 4: (a) temperature contour with along with isolines of zero value of the axial velocity (b) axial velocity contour with streamline at different swirling numbers

In this section the effect of swirling number on flow field characteristics and thermoacoustic oscillations are investigated. $\mathrm{S}_{\mathrm{N}}=0.34,0.45$ and 0.6 , respectively. Fig. 4(a) shows temperature contours along with iso-lines of zero axial velocity. It can be seen that swirling flames shape is V-type. Both corner recirculation zone (CRZ) and central toroidal recirculation zone (CTRZ) are overserved. The CRZ is formed by the sudden expansion from inlet to combustor. As the swirling number is increased, CRZ moves to upstream, which is the result of the high tangential velocity that causes the reattachment point to move upstream. At the same time, the CTRZ also moves upstream. Flames stabilized by both CRZ and CTRZ diverges more toward the radial direction. Axial velocity distributions and streamlines are presented in Fig. 4(b). Wake recirculation zone (WRZ) behind the center rod can be observed at $\mathrm{S}_{\mathrm{N}}=$ 0.34 , however, disappears at the higher swirling number. 

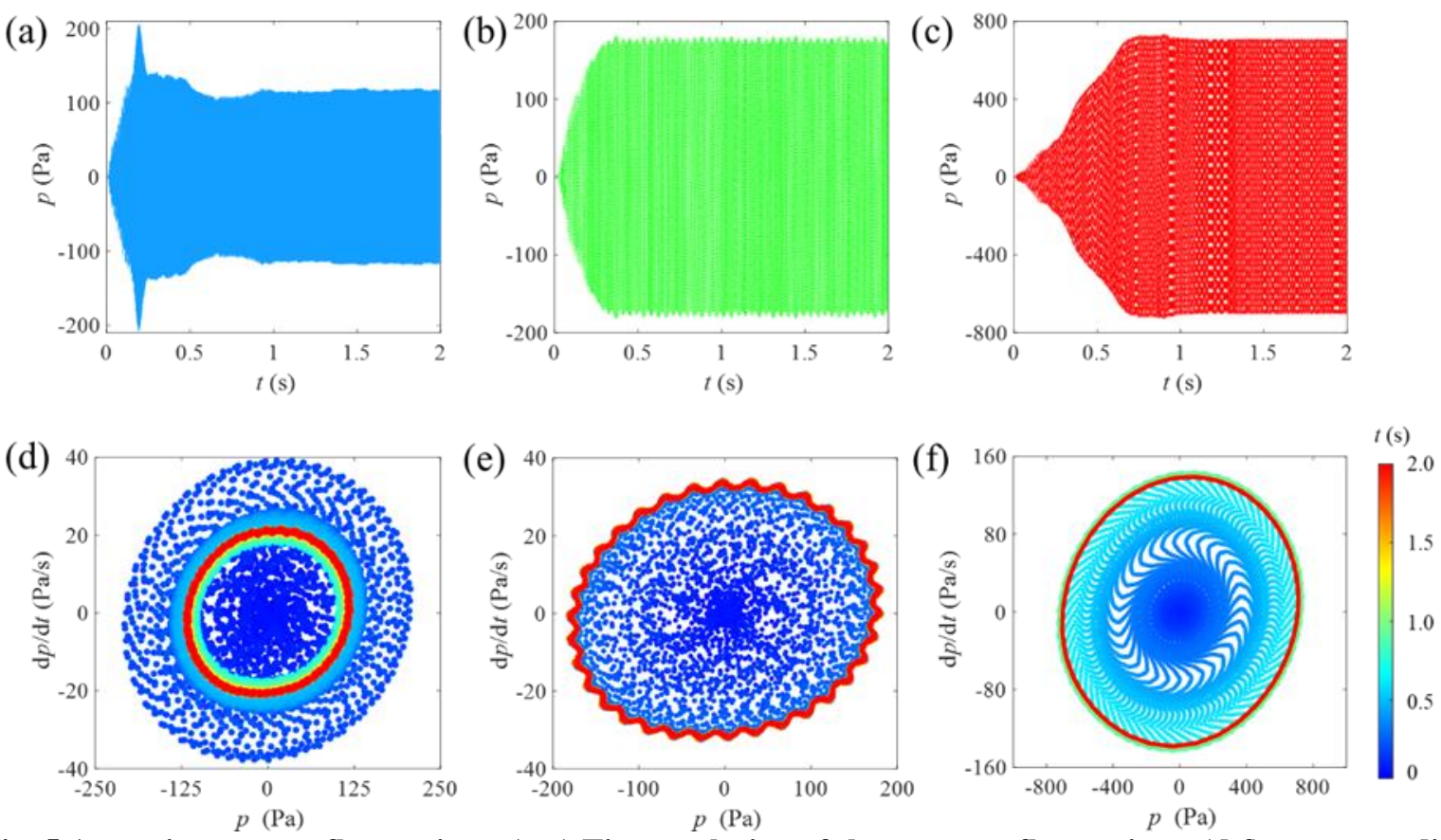

Fig. 5 Acoustic pressure fluctuations. (a-c) Time evolution of the pressure fluctuations, (d-f) corresponding phase diagram of the pressure fluctuations (a), (d) $S_{N}=0.34$, (b), (e) $S_{N}=0.45$, (c), (f) $S_{N}=0.60$

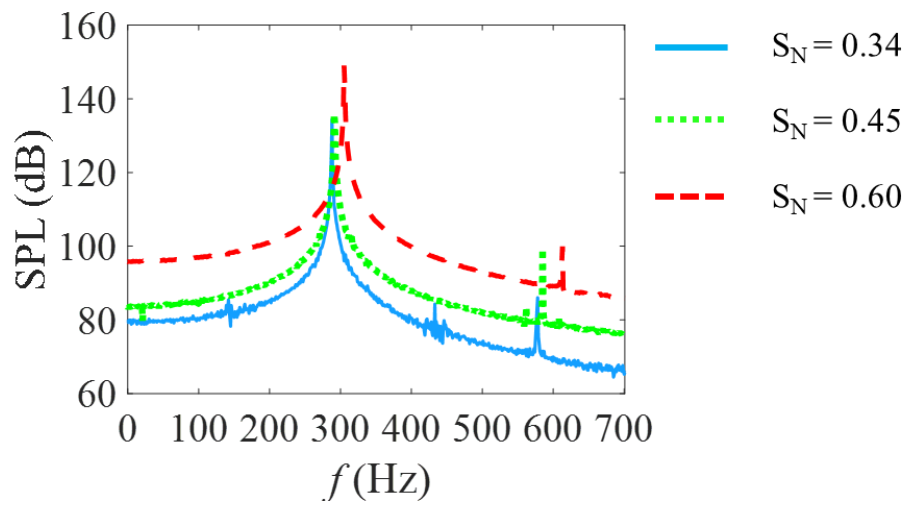

Fig. 6 Frequency spectrum of acoustic pressure fluctuation at different swirling number

The effect of the swirling number on the acoustic pressure perturbation excited by swirling flame is further examined. Fig. 5(a-c) shows the acoustic pressure fluctuations signal in time domain at the $x=328 \mathrm{~mm}$ of the combustor centerline in the axial direction. The amplitude of pressure grows from only a small disturbance initially into a limit cycle. Fig. 5(d-f) shows the corresponding phase plot of the pressure signal at three different swirling numbers. It can be seen that a limit cycles are produced at three conditions. However, the amplitude is increased with increase of swirling number and reach maximum value around $710 \mathrm{~Pa}$ at $\mathrm{S}_{\mathrm{N}}=0.6$. Fig. 6 shows the corresponding frequency spectrum analysis of the pressure signal. The dominant frequency of pressure oscillation is shifted to higher frequency with the increase of swirling number, from $290 \mathrm{~Hz}$ at $\mathrm{S}_{\mathrm{N}}=0.34$ to $307 \mathrm{~Hz}$ at $\mathrm{S}_{\mathrm{N}}=0.6$.

\section{Further Investigation on Heat Exchanger Effect}

In order to achieve an alternative control method of combustion instability, a heat exchanger is wrapped around at a pre-selected segment of combustor wall. Pressure fluctuations in time domain at the plane of $x=328 \mathrm{~mm}$ as $\mathrm{T}_{\mathrm{H}}$ is increased from $500 \mathrm{~K}$ to $1800 \mathrm{~K}$ at $t=2 \mathrm{~s}$ is shown in Fig. 7(a). The limit cycle oscillations in combustor is successfully mitigated by increasing the heat exchanger temperature. It can be seen that when $t \leqslant 2 \mathrm{~s}, \mathrm{~T}_{\mathrm{H}}=500 \mathrm{~K}$, pressure fluctuation 
grows quickly to a limit cycle and be stable at around $t=1.2 \mathrm{~s}$. Here the amplitude of perturbation reaches to about 1550 pa. Then the temperature of heat exchanger is increase to $\mathrm{T}_{\mathrm{H}}=1800 \mathrm{~K}$ at $t=2 \mathrm{~s}$. The amplitude of pressure fluctuation drops gradually and be stable at 215 pa. Fig. 7(b) shows the corresponding sound energy per unit volume in time domain. Sound energy per unit volume $\mathrm{E}_{a}(t)$ can be calculated as [15]:

$$
E_{a}=\frac{1}{T} \frac{\int_{T}\left(p^{\prime}\right)^{2} d t}{2 \gamma p 0}
$$

in the equation, $p^{\prime}$ is the acoustic pressure perturbation, $p_{0}=101325 \mathrm{~Pa}$ is the reference pressure, and $\gamma=1.4$ is the adiabatic index. Increasing $T_{H}$ lead an over $90 \%$ acoustic energy reduction. Fig. 8 shows corresponding spectrum of pressure perturbation before and after $\mathrm{T}_{\mathrm{H}}$ is increased. After $\mathrm{T}_{\mathrm{H}}$ is increased the sound pressure level (SPL) is decreased from $157 \mathrm{~dB}$ to $139.6 \mathrm{~dB}$. At $\mathrm{T}_{\mathrm{H}}=1800 \mathrm{~K}$ case, a local peak at the harmonic frequency $590 \mathrm{~Hz}$ is observed clearly which means the nonlinearity characteristics of the system.
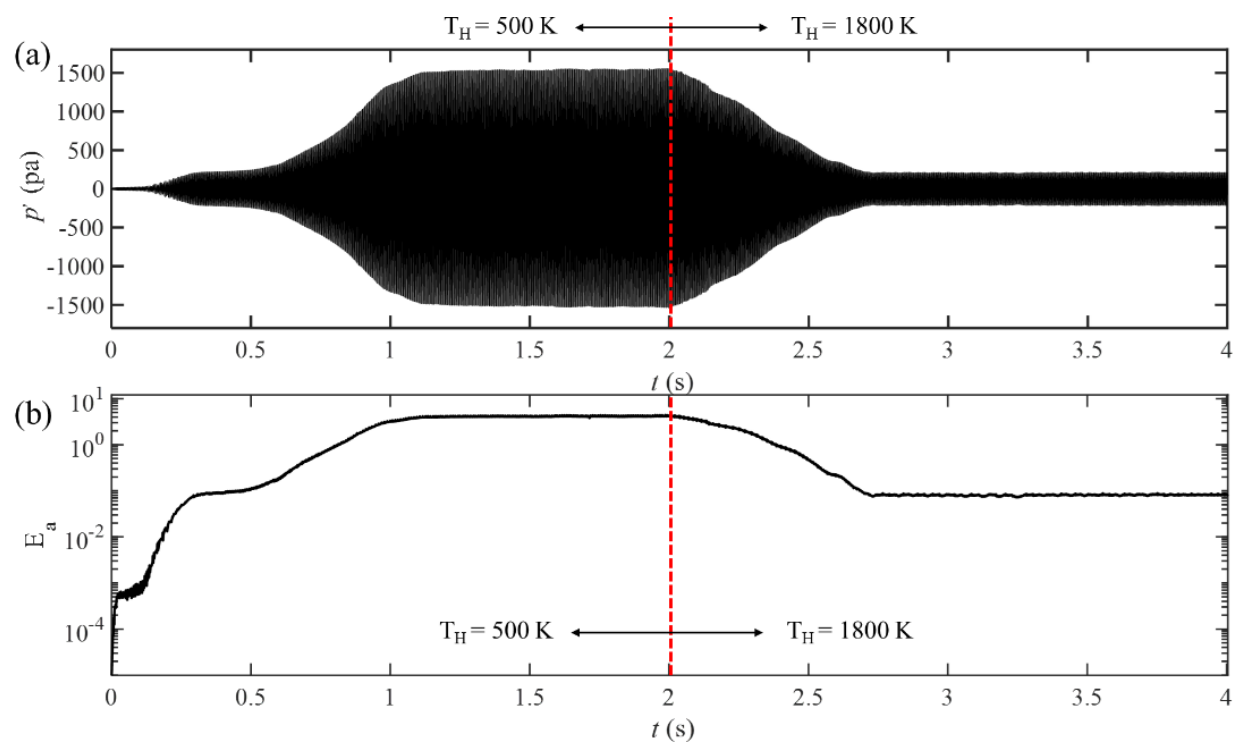

Fig.7 Time evolution of (a) acoustic pressure (b) acoustic energy at $x=328 \mathrm{~mm}$.
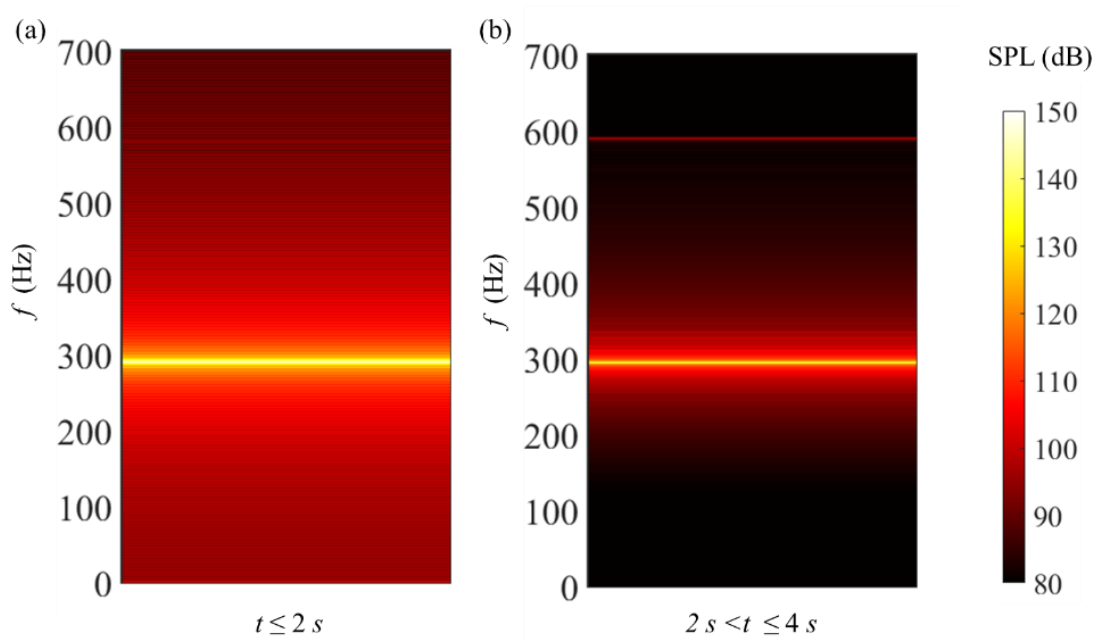

6

American Institute of Aeronautics and Astronautics 
Fig.8 Corresponding spectrum of acoustic pressure at $x=328 \mathrm{~mm}$ (a) $T_{\mathrm{H}}=500 \mathrm{~K}$ (b) $T_{\mathrm{H}}=1800 \mathrm{~K}$
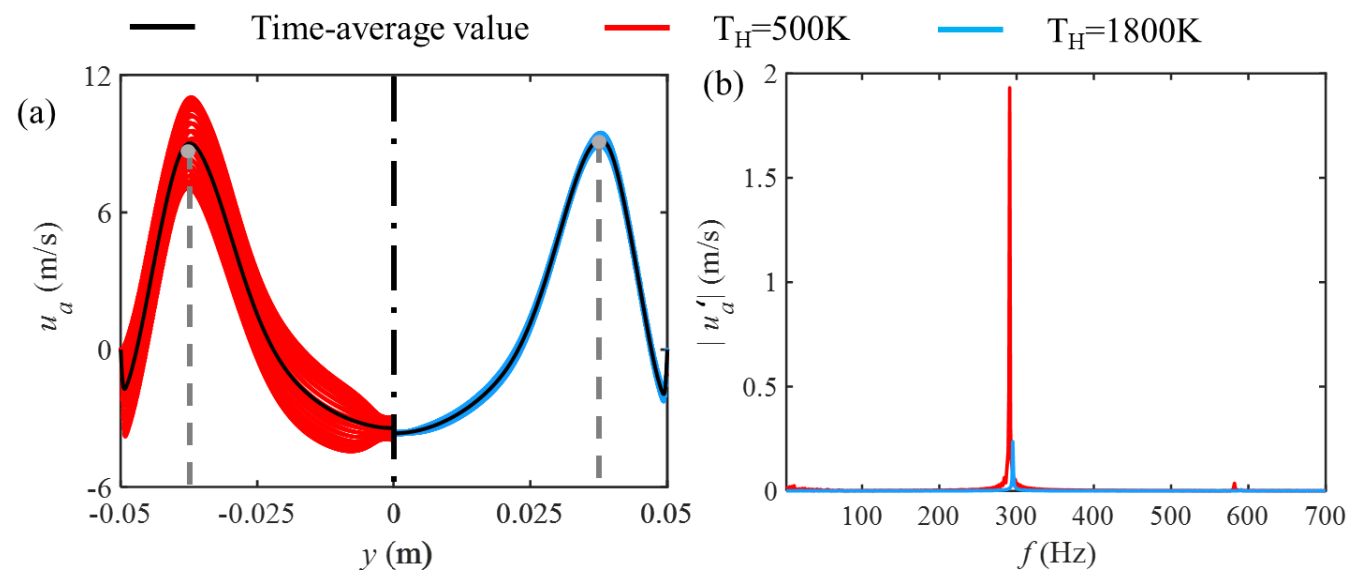

Fig. 9 (a) Axial velocity profile over a period for $T_{H}=500 \mathrm{~K}$ (left) and $T_{H}=1800 \mathrm{~K}$ (right) at $x / R_{\mathrm{c}}=1.2$ (b) corresponding frequency spectrum of the max axial velocity point

Fig. 9(a) shows the variation of axial velocity profile over a period at $\mathrm{T}_{\mathrm{H}}=500 \mathrm{~K}$ and $\mathrm{T}_{\mathrm{H}}=1800 \mathrm{~K}$ at $x / R_{\mathrm{c}}=1$.2. It can be seen that axial velocity fluctuation range at $\mathrm{T}_{\mathrm{H}}=500 \mathrm{~K}$ are much wider that at $\mathrm{T}_{\mathrm{H}}=1800 \mathrm{~K}$. Fig. 9 (b) presents the corresponding frequency spectrum of the axial velocity fluctuation of the max axial velocity point, before and after the $\mathrm{T}_{\mathrm{H}}$ is increased. Around $1.4 \%$ of dominant frequency shifting from $291 \mathrm{~Hz}$ to $295 \mathrm{~Hz}$ is observed and the amplitude of axial velocity perturbation is reduced over $90 \%$ from $\mathrm{T}_{\mathrm{H}}=500 \mathrm{~K}$ to $1800 \mathrm{~K}$.

\section{Conclusions}

In this work the nonlinear self-sustained thermoacoustic oscillations in a swirling combustor are numerical investigated by 3D RANS using the commercial software Ansys Fluent 19.1. Turbulence is modelled by a RNG $k-\varepsilon$ turbulence model and chemistry reaction is modelled using a reduced 2-step combustion kinetic mechanism. The numerical model is firstly validated by experimental data in literature. Then the effect of swirling number $S_{\mathrm{N}}$ on time average combustion and flow field characteristics is examined. At lower swirling number $S_{N}=0.34$, wake recirculation zone (WRZ) behind the central rod can be observed. As $S_{N}$ is increased WRZ disappears, the central toroidal recirculation zone (CTRZ) and corner recirculation zone (CRZ) move to upstream. $\mathrm{S}_{\mathrm{N}}$ determines not only the amplitude but dominant frequency of pulsating acoustic perturbation. As $S_{N}$ is increased the amplitude of pressure is also increased. Increasing $S_{N}$ from 0.34 to 0.6 leads an approximately $6 \%$ of the dominant frequency shift. Furthermore, an alternative control approach to attenuate combustion instability by implementing a heat exchanger at a pre-selected segment of combustor wall $\mathrm{T}_{\mathrm{H}}$ is applied and studied. Oscillations excited by the swirling flame in combustor is successfully damped by increasing the heat exchanger temperature $\mathrm{T}_{\mathrm{H}}$. Amplitude, acoustic energy and axial velocity fluctuation range are reduced after increasing $\mathrm{T}_{\mathrm{H}}$. Dominant frequency of pressure and axial velocity spectrum are found a $1.6 \%$ shift to higher frequency. A clear harmonic frequency peak is shown in pressure spectrum, which means the system is nonlinear after increasing $\mathrm{T}_{\mathrm{H}}$. The present study contributes to developing a platform to conduct numerical study of nonlinear thermoacoustic oscillation in swirling combustors. It also opens up an alternative control method to damp the large amplitude thermoacoustic instabilities.

\section{Acknowledgement}

This work was financially supported by the University of Canterbury, New Zealand with Grant No. 452STUPDZ, and National Research Foundation, Prime Minister's Office, Singapore, with Grant No. NRF2016NRF-NSFC001102 and National Natural Science Foundation of China (11661141020).

\section{References}

1. Lieuwen, T., and McManus, K. "Introduction: Combustion dynamics in lean-premixed prevaporized (LPP) gas turbines," Journal of Propulsion and Power Vol. 19, No. 5, 2003, pp. 721-721. 
2. Keller, J. J. "Thermoacoustic oscillations in combustion chambers of gas turbines," AIAA journal Vol. 33, No. 12, 1995, pp. 2280-2287.

3. Li, H., Zhou, X., Jeffries, J. B., and Hanson, R. K. "Sensing and control of combustion instabilities in swirl-stabilized combustors using diode-laser absorption," AIAA journal Vol. 45, No. 2, 2007, pp. 390-398.

4. Steinberg, A. M., Boxx, I., Stohr, M., Meier, W., and Carter, C. D. "Effects of flow structure dynamics on thermoacoustic instabilities in swirl-stabilized combustion," AIAA journal Vol. 50, No. 4, 2012, pp. 952-967.

5. Huang, Y., and Yang, V. "Effect of swirl on combustion dynamics in a lean-premixed swirl-stabilized combustor," Proceedings of the Combustion Institute Vol. 30, 2005, pp. 1775-1782.

6. Yellugari, K., Villalva Gomez, R., and Gutmark, E. J. "Effects of Swirl Number and Central Rod on Flow in Lean Premixed Swirl Combustor," AIAA Scitech 2020 Forum. 2020, p. 0433.

7. Mansouri, Z., Aouissi, M., and Boushaki, T. "A numerical study of swirl effects on the flow and flame dynamics in a lean premixed combustor," International journal of heat and technology Vol. 34, No. 2, 2016, pp. 227-235.

8. DeLaat, J. C., Kopasakis, G., Saus, J. R., Chang, C. T., and Wey, C. "Active combustion control for a low-emissions aircraft engine combustor prototype: Experimental results," Journal of Propulsion and Power Vol. 29, No. 4, 2013, pp. 991-1000.

9. Gelbert, G., Moeck, J. P., Paschereit, C. O., and King, R. "Feedback control of unstable thermoacoustic modes in an annular Rijke tube," Control Engineering Practice Vol. 20, No. 8, 2012, pp. 770-782.

10. Li, S., Li, Q., Tang, L., Yang, B., Fu, J., Clarke, C. A., Jin, X., Ji, C. Z., and Zhao, H. "Theoretical and experimental demonstration of minimizing self-excited thermoacoustic oscillations by applying anti-sound technique," Applied Energy Vol. 181, 2016, pp. 399-407.

11. Wu, G., Lu, Z., Pan, W., Guan, Y., Li, S., and Ji, C. Z. "Experimental demonstration of mitigating self-excited combustion oscillations using an electrical heater," Applied Energy Vol. 239, 2019, pp. 331-342.

12. Sun, Y., Rao, Z., Zhao, D., Wang, B., Sun, D., and Sun, X. "Characterizing nonlinear dynamic features of self-sustained thermoacoustic oscillations in a premixed swirling combustor," Applied Energy Vol. 264, 2020, p. 114698.

13. Huang, Y., and Yang, V. "Dynamics and stability of lean-premixed swirl-stabilized combustion," Progress in Energy and Combustion Science Vol. 35, No. 4, 2009, pp. 293-364.

14. Taamallah, S., Dagan, Y., Chakroun, N., Shanbhogue, S., Vogiatzaki, K., and Ghoniem, A. F. "Helical vortex core dynamics and flame interaction in turbulent premixed swirl combustion: A combined experimental and large eddy simulation investigation," Physics of Fluids Vol. 31, No. 2, 2019, p. 025108.

15. Zhao, D., Guan, Y., and Reinecke, A. "Characterizing hydrogen-fuelled pulsating combustion on thermodynamic properties of a combustor," Communications Physics Vol. 2, No. 1, 2019. 